\title{
Multiple Step Interlaced Beam Scan to Minimize the Deviation of Radar Detection Performance
}

\author{
Ji Hwan Yoon $\cdot$ Yeonhee Park · Ji Eun Roh · Sung Chul Park
}

\begin{abstract}
For detection of targets, a radar conventionally scans a specified search volume with a fixed beam lattice of a specified beam spacing. With the fixed beam lattice, the detection performance of the radar within a unit beam lattice changes depending on the line-of-sight angle to a target. In this paper, multiple step interlaced scan is proposed to minimize the deviation of the detection performance due to the change of the target line-of-sight angle. As a figure of merit for the detection performance, the cumulative probability of detection is analyzed for various values of the interlaced scan step with different beam overlap ratios, and the optimal values of the steps to minimize the deviation of the cumulative probability of detection are derived.
\end{abstract}

Key Words: Beam Lattice, Cumulative Probability of Detection, Interlaced Scan, Phased-Array Radar, Search Radar.

\section{INTRODUCTION}

One of the major roles of a phased-array radar is to detect targets by scanning a specified search volume. The radar attempts to detect the targets by stepping beams in the search volume with a pre-defined beam lattice. The beam lattice can be formed either in a rectangular or a triangular array [1]. The total number of the beams to scan the given search volume is determined by the spacing between the adjacent beams. As the beam spacing increases, the total number of the beams decreases. This results in the reduction of a scan time, but the cost is increase of a beamshape loss [2-4]. The beamshape loss is introduced because the target is not always at the center of the beam. When the line-of-sight (LOS) angle to the target is off from the beam center, the strength of the target echo is reduced depending on the antenna beam pattern. Therefore, the beam spacing should be determined based on the trade-off between the scan time and the beamshape loss.

As a radar scans with a fixed beam lattice for the specified beam spacing, the detection performance of the radar can vary drastically depending on the LOS angle to a target, and is significantly reduced when the target is unfortunately in the middle of two adjacent beam positions. In this paper, interlaced scan with multiple steps is proposed to reduce such deviation of the detection performance. As a figure of merit for the detection performance, cumulative probability of detection is analyzed for the different steps of the interlaced scan. Then, the optimal values of the interlaced scan step to minimize the deviation are derived for different values of beam spacing.

This paper is organized as follows. In Section II, the concept of the multiple step interlaced scan is presented. In Section III, analysis methods to compare the detection performances with different steps of interlaced scan are explained. The analysis results are provided in Section IV, and Section V is the con-

Manuscript received August 18, 2019 ; Revised December 20, 2019 ; Accepted February 6, 2020. (ID No. 20190818-067J)

Agency for Defense Development, Daejeon, Korea.

"Corresponding Author: Ji Hwan Yoon (e-mail: saijy4@add.re.kr)

This is an Open-Access article distributed under the terms of the Creative Commons Attribution Non-Commercial License (http://creativecommons.org/licenses/by-nc/4.0) which permits unrestricted non-commercial use, distribution, and reproduction in any medium, provided the original work is properly cited.

(c) Copyright The Korean Institute of Electromagnetic Engineering and Science. All Rights Reserved. 
clusion.

\section{MULTIPLE STEP INTERLACED SCAN}

In Fig. 1, a configuration of the multiple step interlaced scan is shown with $N_{s}$ steps. The basic beam lattice considered in this work is designed with a beam spacing $\rho \Delta u$, where $\rho$ is the beam overlap ratio $(\rho>0)$ and $\Delta u$ is the 1-way 3-dB beamwidth of the antenna in sine space (It is preferable to design a beam lattice in sine space, since the beamwidth does not change with scan angle in sine space [5]). The circles of each scan in Fig. 1 represent the 3-dB beamwidth contours of the beams.

With conventional search beam scanning, the beam positions are fixed for each scan. With multiple step interlaced scan proposed in this work, however, the beam positions in the next scan are shifted from the positions of the previous scan. For $N_{s}$ step interlaced scan, the amount of the shift is $\rho \Delta u / N_{s}$. In Fig. 1, the beam positions of the $2^{\text {nd }}$ scan are shifted by $\rho \Delta u / N_{s}$ from those of the $1^{\text {st }}$ scan. In case of $N_{s}>2$, the beam positions of the $3^{\text {rd }}$ scan are also shifted by $\rho \Delta u / N_{s}$ from those of the $2^{\text {nd }}$ scan. Consequently, the beam positions of the $N_{s}^{\text {th }}$ scan would be shifted by $\frac{\rho \Delta u\left(N_{s}-1\right)}{N_{s}}$ from those of the $1^{\text {st }}$ scan. After the $N_{s}^{\text {th }}$ scan is finished, the scan restarts with the beam positions of the $1^{\text {st }}$ scan, and the same sequence is repeated.

In [6], the interlaced scan with $N_{s}=2$ was introduced to mitigate the effect of beamshape loss. However, the interlaced scan with $N_{s}=2$ does not always guarantee the minimum deviation of the detection performance, depending on the target LOS angle. Therefore, in this work, the concept of the interlaced scan is extended to $N_{s} \geq 2$ to minimize such deviation. Specifically, in Section IV, it is shown that $N_{s}$ must be increased to minimize the deviation as $\rho$ increases and the optimum $N_{s}$ for various

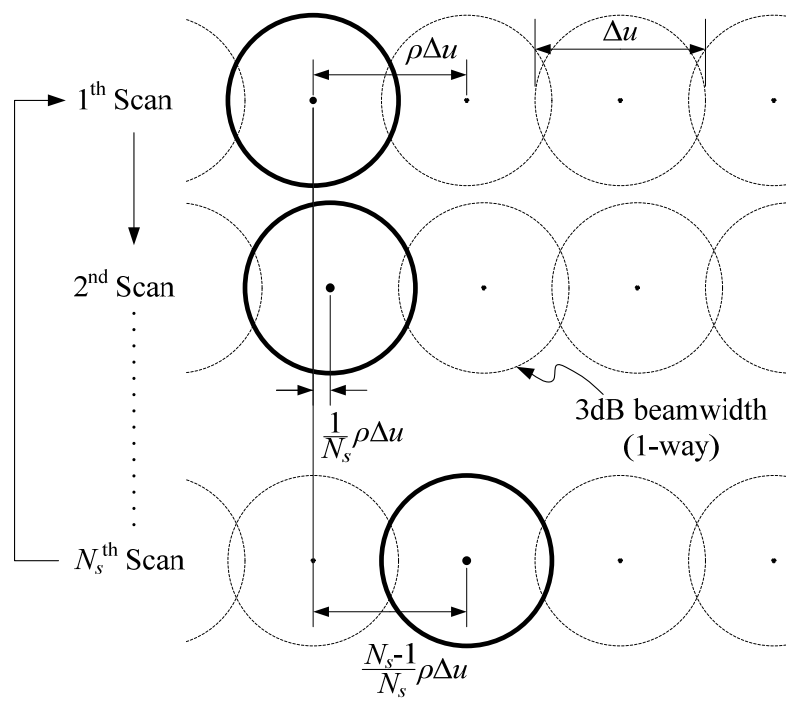

Fig. 1. Beam lattices for the multiple step interlaced scan with $N_{s}$ steps. ranges of $\rho$ is provided.

Although the multiple step interlaced scan can also be applied in elevation, in this work, it is applied only in azimuth for simplicity.

\section{ANALYSIS METHOD OF DETECTION PERFORMANCE}

In this section, the analysis methods of the radar detection performance are presented. First, the antenna beam pattern model for the beam lattice design and the calculation of the beamshape loss are described. Second, the calculation for the cumulative probability of detection as a figure of merit of the radar detection performance is explained. Third, a scenario for the analysis of the detection performance is described.

\section{Antenna Beam Pattern Model}

To analyze the effect of the multiple step interlaced scan on the detection performance, a loss of signal-to-noise ratio (SNR) when the target is off from the beam center needs to be calculated. This beamshape loss is the reduction of the 2-way antenna gain used in radar equation, and can be calculated from the antenna beam pattern. In this work, the antenna beam pattern $F(\theta)$ is modelled with a cosine-illuminated pattern as follows [1]:

$$
F(\theta)=\frac{\cos (\pi D \sin (\theta) / \lambda)}{1-(2 D \sin (\theta) / \lambda)^{2}}
$$

where $D$ is the largest dimension of the antenna aperture, $\theta$ is the angle from the antenna boresight, and $\lambda$ is the wavelength of the radar signal. In this work, it is assumed that $D=34 \lambda$ which is a reasonable value for radar application that requires high directivity. In this case, the 1 -way $3 \mathrm{~dB}$ beamwidth $\theta_{3 \mathrm{~dB}}$ is $2^{\circ}$, and therefore, $\Delta u=\sin \left(\theta_{3 \mathrm{~dB}}\right) \approx 0.035$. Note that the exact value of the beamwidth is not important in the analysis of the proposed multi-step interlaced scan proposed in this work, as the optimal $N_{s}$ depends on the beam overlap ratio $\rho$, not on the beamwidth itself.

When the target is off from the antenna beam center by $\theta_{t}$ at a single beam, the beamshape loss is $1 / F^{2}\left(\theta_{t}\right)$ as shown in Fig. 2 .

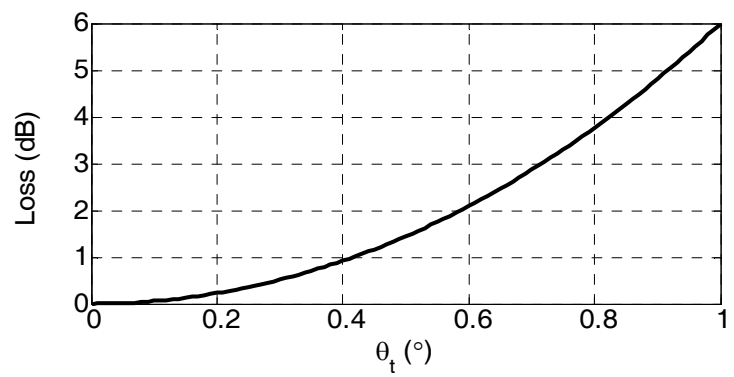

Fig. 2. The beamshape loss of the radar against the target LOS angle from the beam center $\left(\theta_{t}\right)$. 


\section{Probability of Detection Calculation}

Detection performance of a radar is usually given as its detection range at a specified cumulative probability of detection. The cumulative probability of detection at a range $R$ is the probability that the radar will detect an approaching target at least once by the time the target reaches $R$ [7]. A simple expression of the cumulative probability of detection at the $n^{\text {th }}$ scan $P_{c}(n)$ is given by

$$
P_{c}(n)=1-\prod_{m=1}^{n}\left[1-P_{d s}(m)\right]
$$

where $P_{d s}(m)$ is a single probability of detection at $m^{\text {th }}$ scan.

The single probability of detection is a probability that the target will be detected in a single search scan. The target can be detected by more than one beam in the search scan. If there are $k$ beams in a single scan, $P_{d s}(m)$ is given by

$$
P_{d s}(m)=1-\prod_{i=1}^{k}\left[1-P_{d}(i, m)\right]
$$

where $P_{d}(i, m)$ is a single probability of detection at the $i^{\text {th }}$ beam of the $m^{\text {th }}$ scan. Note that, in this work, the detections of the beams are assumed to be uncorrelated for the calculation of $P_{d s}$.

The target fluctuation model for the calculation of the probability of detection is assumed as Swerling case 1. Therefore, for a single beam, $P_{d}$ is given as

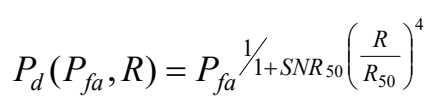

where $P_{f a}$ is the probability of false alarm. $S N R_{50}$ and $R_{50}$ are target echo SNR and range where $P_{d}=50 \%$, respectively.

\section{Scenario}

To analyze the effect of the multiple step interlaced scan on the radar detection performance, the following scenario is considered. The radar scans the search volume of $\pm 60^{\circ}$ in azimuth with one elevation bar. The target approaches to the radar from the maximum range of $R_{\max }=300 \mathrm{~km}$ with a constant velocity of $V_{t}=500 \mathrm{~m} / \mathrm{s}$. In the calculation of the probability of detection, it is assumed that $R_{50}=100 \mathrm{~km}, S N R_{50}=12.8 \mathrm{~dB}$, and $P_{f a}$ $=10^{-6}$.

The target LOS angle is fixed as the target approaches to the radar. To show how the multiple step interlaced scan reduce the deviation of the detection performance due to the target LOS

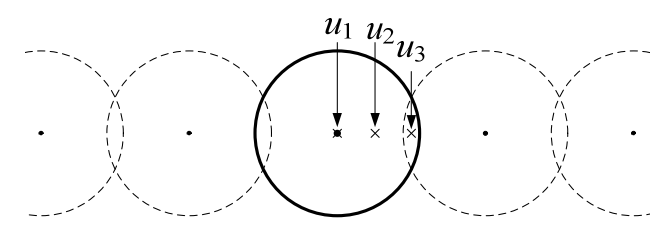

Fig. 3. The three LOS angles of the target. angle change, three LOS angles of the target are considered as shown in Fig. 3. The three LOS angles $u_{d}(d=1,2,3)$ are uniformly spaced from 0 to the half of the beam spacing. Therefore,

$$
u_{d}=\frac{(d-1) \rho \Delta u}{4} .
$$

\section{ANALYSIS RESULTS}

In this section, the cumulative probability of detection is calculated for the different values of $\rho$ with the previously described scenario. For each $\rho$, the deviation of the cumulative probability of detection is compared for the different values of $N_{s}$, and the optimal $N_{s}$ to minimize the deviation is derived.

\section{Case for $\rho=1.0$}

Fig. 4 shows $P_{d s}$ and $P_{c}$ for the different steps of the interlaced scan when $\rho=1.0$. Fig. $4(\mathrm{a})$ is with $N_{s}=1$, i.e., no interlaced scan. It is shown that, for the same range, $P_{c}$ is reduced as $u_{d}$ increases (or in other words, as the target LOS angular distance from beam center increases). This is because the SNR is reduced due to the increase of the beamshape loss. There is no beamshape loss for the target echo signal at $u_{1}$, but the beamshape loss at $u_{3}$ is $6 \mathrm{~dB}$. The range for $P_{c}=90 \%$ is decreased from $130 \mathrm{~km}$ to $96 \mathrm{~km}$ as $u_{d}$ changes from $u_{1}$ to $u_{3}$. This is not desirable, since the detection performance of the radar varies drastically depending on the target LOS angles, and is significantly degraded when the target is unfortunately close to the middle of two adjacent beam positions.

By introducing the interlaced scan, the deviation of $P_{c}$ at the same $R$ is reduced and unexpected degradation of the detection performance can be avoided. Fig. 4(b) shows $P_{d s}$ and $P_{c}$ with $N_{s}$ $=2$. As the beam positions are interlaced between the consecutive search scans, the beamshape loss is averaged among different target LOS angles. Note that $P_{d s}$ curves show fluctuation where the period of the fluctuation is $N_{s}$ times the single search scan time. The fluctuation is due to the change of the beamshape loss as the beam positions in each search scan is shifted by interlacing. In case of $N_{s}=1$, no fluctuation of $P_{d s}$ is observed since the beam positions are always fixed. As the $P_{d s}$ curves fluctuate due to the effect of averaged beamshape loss by introducing multi-step interlaced scan, the $P_{d s}$ curves for different LOS angles of the target eventually overlap. Since the $P_{c}$ directly depends on $P_{d s}$, the $P_{c}$ curves also overlaps. In other words, the deviation of $P_{c}$ is reduced.

With $N_{s}=3$, the deviation can be further reduced, as shown in Fig. 4(c). However, higher $N_{s}$ does not always guarantee lower level of deviation. Fig. 4(d) shows $P_{c}$ with $N_{s}=10$ and it is observed that the level of deviation is increased. This is because too dense steps of interlacing (too large $N_{s}$ ) makes the target to be visited by beams with similar positions (thus, similar level of 


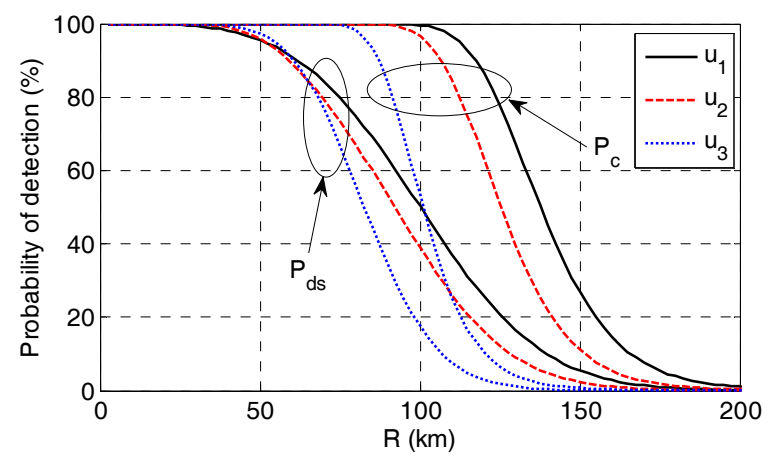

(a)

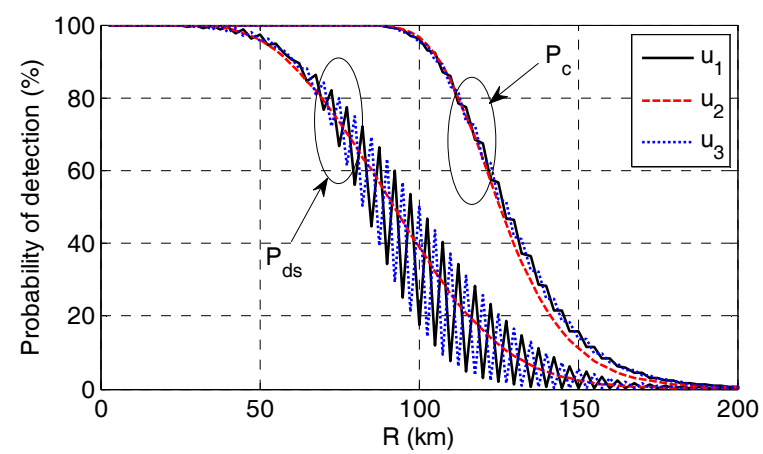

(b)

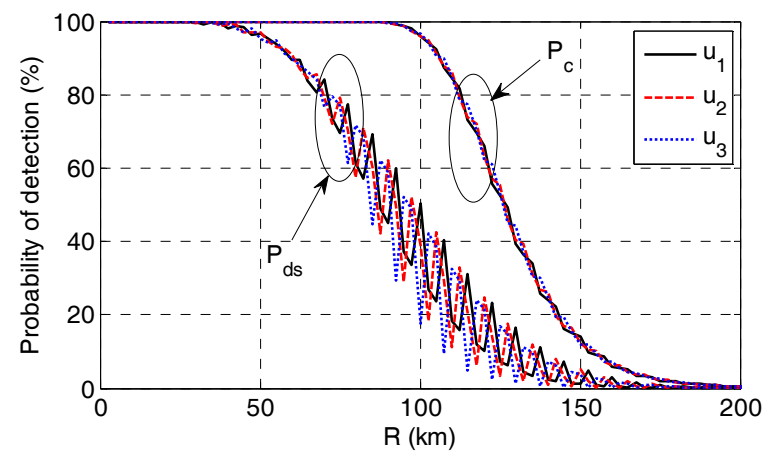

(c)

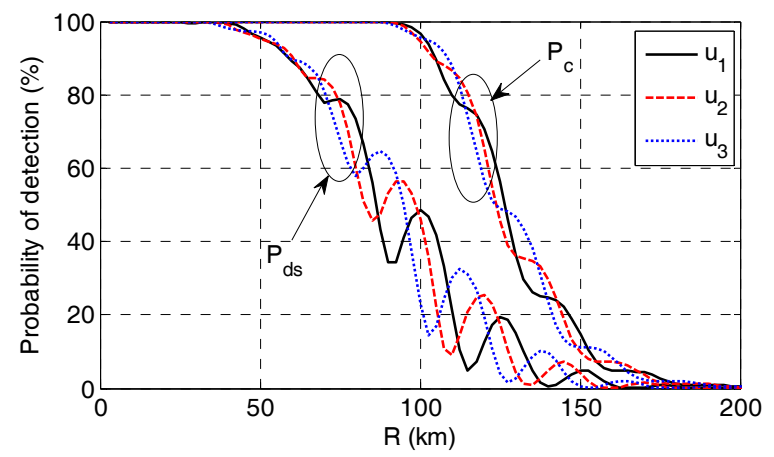

(d)

Fig. 4. The probability of detection with $\rho=1.0$ : (a) no interlaced $\operatorname{scan}\left(N_{s}=1\right),\left(\right.$ b) $N_{s}=2$, (c) $N_{s}=3$, and (d) $N_{s}=10$.

beamshape loss) for undesirably long time. In other words, for a given $\rho$, there is optimal $N_{s}$ that can minimize the deviation of the cumulative probability of detection.

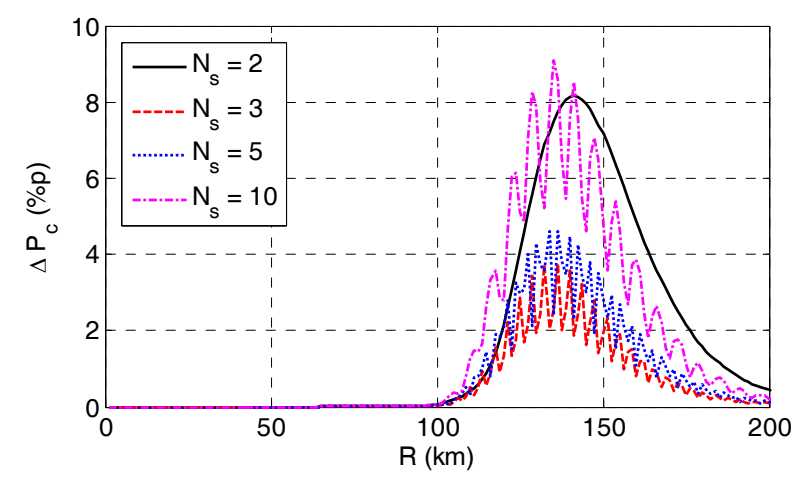

Fig. 5. Deviation of cumulative probability of detection with $\rho=$ 1.0 .

The level of deviation can be analyzed by comparing the highest $P_{c}$ and the lowest $P_{c}$ among the three target LOS angles in the same range. In Fig. 5, $\Delta P_{c}$ (the difference between the highest $P_{c}$ and the lowest $P_{c}$ ), are shown for the different $N_{s}$. For $N_{s}=2, P_{c}$ can vary up to $8.1 \% \mathrm{p}$ at $R=141.3 \mathrm{~km}$ (where $\Delta P_{c}=$ $1.9 \% \mathrm{p}$ for $N_{s}=3, \Delta P_{c}=2.3 \% \mathrm{p}$ for $N_{s}=5$, and $\Delta P_{c}=1.9 \% \mathrm{p}$ for $N_{s}=10$ ), but for $N_{s}=3$, the maximum $\Delta P_{c}$ is only 3.8\%p at $R=$ $136.3 \mathrm{~km}$ (where $\Delta P_{c}=7.8 \% \mathrm{p}$ for $N_{s}=2, \Delta P_{c}=4.6 \% \mathrm{p}$ for $N_{s}=$ 5 , and $\Delta P_{c}=8.6 \% \mathrm{p}$ for $\left.N_{s}=10\right)$. For $N_{s}=5$, the maximum $\Delta P_{c}$ is increased to $4.6 \% \mathrm{p}$ at $R=136.3 \mathrm{~km}$ (where $\Delta P_{c}=7.8 \% \mathrm{p}$ for $N_{s}=2, \Delta P_{c}=3.8 \% \mathrm{p}$ for $N_{s}=3$, and $\Delta P_{c}=8.6 \% \mathrm{p}$ for $N_{s}=10$ ). For $N_{s}=10$, the maximum $\Delta P_{c}$ is increased to $9.1 \%$ p at $R=$ $135.0 \mathrm{~km}$ (where $\Delta P_{c}=7.5 \% \mathrm{p}$ for $N_{s}=2, \Delta P_{c}=2.6 \% \mathrm{p}$ for $N_{s}=$ 3 , and $\Delta P_{c}=2.4 \% \mathrm{p}$ for $N_{s}=5$ ). The optimum $N_{s}$ is the one where the maximum $\Delta P_{c}$ in the whole range of $R$ is the smallest, which is in this case, $N_{s}=3$.

\section{Case for $\rho=2.0$}

The analysis results for $\rho=2.0$ are shown in Figs. 6 and 7 . Note that in Fig. 6(a) for $N_{s}=1$, the deviation of $P_{c}$ becomes more severe than that of Fig. 4(a) for $\rho=1.0$. This is due to the increased beamshape loss originated from the increased beam spacing. The range for $P_{c}=90 \%$ is decreased from $141.5 \mathrm{~km}$ to $22.0 \mathrm{~km}$ as the target LOS angle changes from $u_{1}$ to $u_{3}$. As in the case with $\rho=1.0$, it is observed that the deviation of $P_{c}$ can be reduced by applying the interlaced scan. For $N_{s}=3, P_{c}$ can vary up to $33.2 \%$ p at $R=135.0 \mathrm{~km}$ (where $\Delta P_{c}=9.0 \%$ p for $N_{s}$ $=4, \Delta P_{c}=3.3 \% \mathrm{p}$ for $N_{s}=5$, and $\Delta P_{c}=6.4 \% \mathrm{p}$ for $\left.N_{s}=10\right)$, but for $N_{s}=4$, the maximum $\Delta P_{c}$ is only $9.6 \%$ p at $R=140.0 \mathrm{~km}$ (where $\Delta P_{c}=30.1 \% \mathrm{p}$ for $N_{s}=3, \Delta P_{c}=4.4 \% \mathrm{p}$ for $N_{s}=5$, and $\Delta P_{c}=6.5 \%$ p for $\left.N_{s}=10\right)$. For $N_{s}=5$, the maximum $\Delta P_{c}$ is decreased to $5.1 \% \mathrm{p}$ at $R=130.6 \mathrm{~km}$ (where $\Delta P_{c}=29.4 \% \mathrm{p}$ for $N_{s}$ $=3, \Delta P_{c}=7.0 \% \mathrm{p}$ for $N_{s}=4$, and $\Delta P_{c}=8.8 \% \mathrm{p}$ for $N_{s}=10$ ). For $N_{s}=10$, the maximum $\Delta P_{c}$ is increased to $9.0 \%$ p at $R=135.6$ $\mathrm{km}$ (where $\Delta P_{c}=29.0 \% \mathrm{p}$ for $N_{s}=3, \Delta P_{c}=8.3 \% \mathrm{p}$ for $N_{s}=4$, and $\Delta P_{c}=4.1 \% \mathrm{p}$ for $N_{s}=5$ ). 


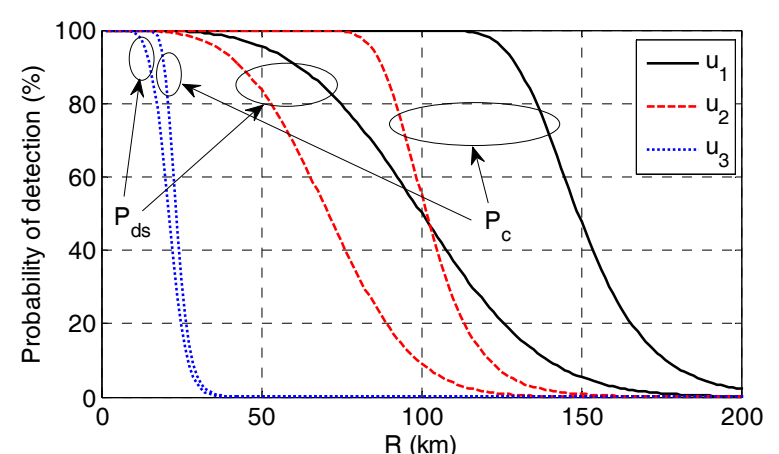

(a)

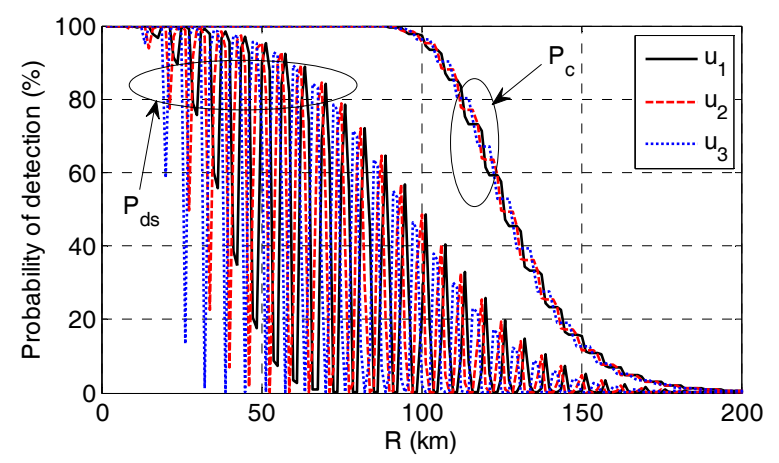

(c)

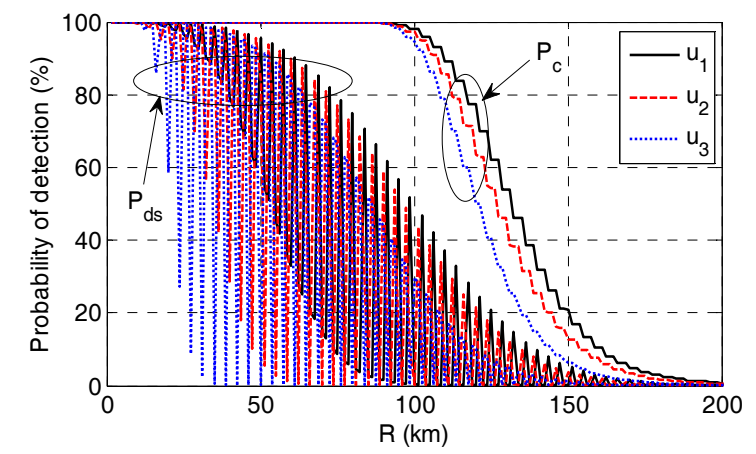

(b)

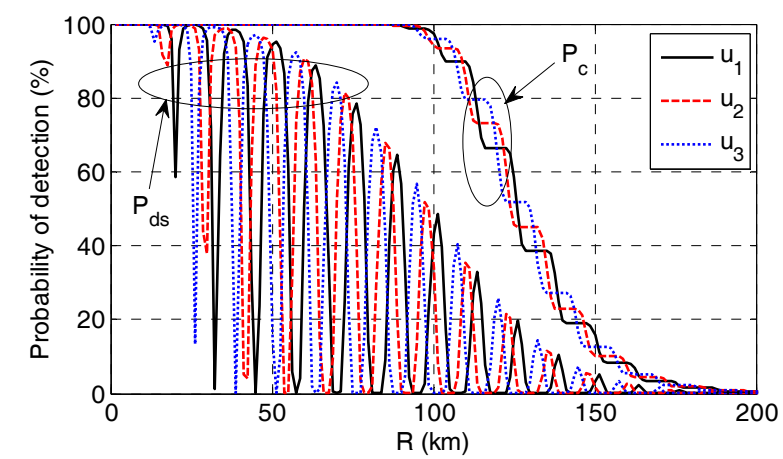

(d)

Fig. 6. The probability of detection with $\rho=2.0$ : (a) no interlaced scan $\left(N_{s}=1\right)$, (b) $N_{s}=3$, (c) $N_{s}=5$, and (d) $N_{s}=10$.

Therefore, in this case, the deviation is minimized when $N_{s}=$ 5 where the maximum $\Delta P_{c}$ is $5.1 \% \mathrm{p}$ at $R=130.6 \mathrm{~km}$, as shown in Fig. 7.

\section{Optimal $N_{s}$}

In Fig. 8, the maximum values of $\Delta P_{c}$ are calculated for $N_{s}$ from 1 to 10 for the different values of $\rho$. It is observed that the optimal $N_{s}$ to minimize the maximum $\Delta P_{c}$ varies depending on $\rho$. In Table 1, the optimal values of $N_{s}$ for the different ranges of $\rho$ are summarized. It is shown that $N_{s}=1$ (the conventional scan with fixed beam lattice) is not optimum for any value of $\rho$, and $N_{s}=2$ is optimum only when $\rho \leq 0.8$. When $\rho>0.8, N_{s}$ needs to be increased to minimize the deviation of $P_{c}$.

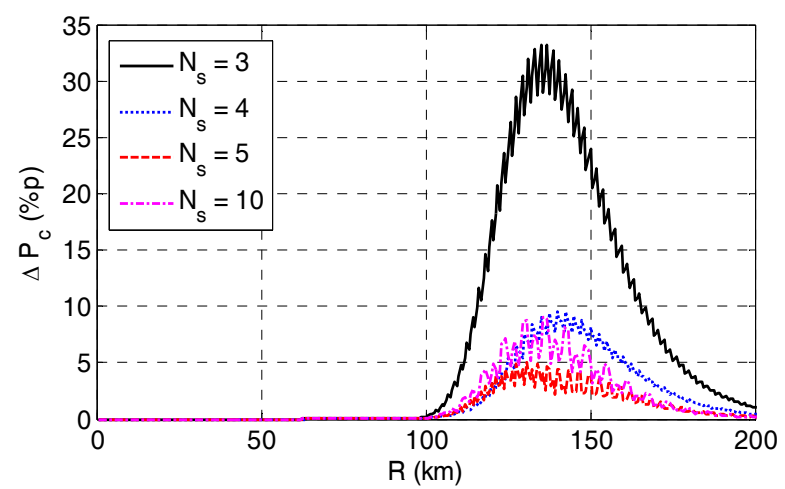

Fig. 7. Deviation of cumulative probability of detection with $\rho=$ 2.0 .

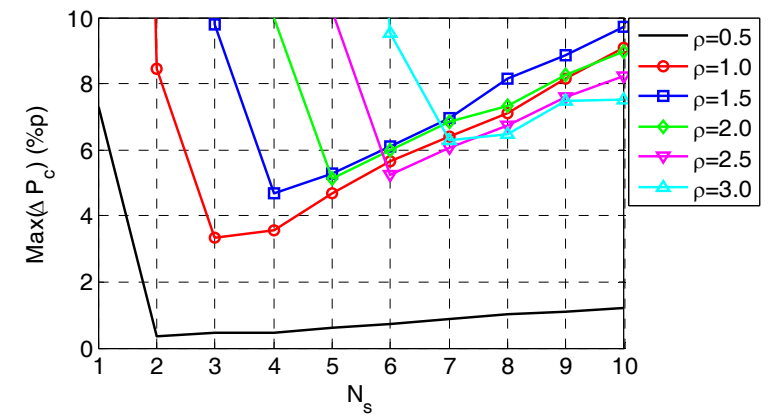

Fig. 8. The maximum deviation of the cumulative probability of detection for the different beam overlap ratios.

Table 1. The optimal $N_{s}$ to minimize the deviation of the cumulative probability of detection for various values of $\rho$

\begin{tabular}{cc}
\hline & $N_{s}$ \\
\hline$\rho \leq 0.8$ & 2 \\
$0.8<\rho \leq 1.2$ & 3 \\
$1.2<\rho \leq 1.7$ & 4 \\
$1.7<\rho \leq 2.1$ & 5 \\
$2.1<\rho \leq 2.6$ & 6 \\
$2.6<\rho \leq 3.0$ & 7 \\
\hline
\end{tabular}

\section{CONCLUSION}

In this work, it has been shown that the deviation of the radar detection performance for different target LOS angle can be 
reduced by applying multiple step interlaced scan. By comparing the cumulative probability of detection with different values of the interlaced scan step, the optimal interlaced scan steps to minimize the deviation of the detection performance have been derived for various beam overlap ratios. By applying the proposed multiple step interlaced scan, it is expected that a radar engineer can design a radar with more stable detection performance.

Although the optimum $N_{s}$ has been derived based on parametric studies of $N_{s}$ and $\rho$, more theoretical analysis methods to derive the optimum $N_{s}$ can be investigated as a future work.

\section{REFERENCES}

[1] J. E. Fielding, "Beam overlap impact on phased-array target detection," IEEE Transactions on Aerospace and Electronic Systems, vol. 29, no. 2, pp. 404-411, 1993.

[2] L. V. Blake, "The effective number of pulses per beamwidth

\section{Ji Hwan Yoon}

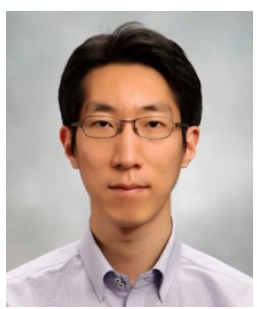

received the Ph.D. degree in Electrical and Electronic Engineering from Yonsei University, South Korea, in 2016. Since March, 2016, he has been with the Agency for Defense and Development, South Korea, where he is currently a senior researcher. His research interests include airborne radar systems, phased array radars, and reflectarrays.

\section{Yeonhee Pak}

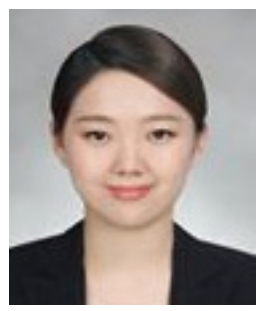

received the M.S. degree in Electrical and Electronic Engineering from Yonsei University, South Korea, in 2016. Since March, 2016, he has been with the Agency for Defense and Development, South Korea, where he is currently a Principal researcher. Her research interests include airborne radar systems.

for a scanning radar," Proceedings of the IRE, vol. 41, no. 6, pp. 770-774, 1953.

[3] P. M. Hahn and S. D. Gross, "Beam shape loss and surveillance optimization for pencil beam arrays," IEEE Transactions on Aerospace and Electronic Systems, vol. 5, no. 4, pp. 674-675, 1969.

[4] W. M. Hall and D. K. Barton, "Antenna pattern loss factor for scanning radars," Proceedings of the IEEE, vol. 53, no. 9, pp. 1257-1258, 1965.

[5] A. D. Brown, Electronically Scanned Arrays MATLAB Modeling and Simulation. Boston, MA: CRC Press, 2012.

[6] E. R. Billam, "The problem of time in phased array radar," in Proceedings of IEE International Radar Conference (RADAR), Edinburgh, UK, 1997, pp. 563-575.

[7] J. D. Mallett and L. E. Brennan, "Cumulative probability of detection for targets approaching a uniformly scanning search radar," Proceedings of the IEEE, vol. 51, no. 4, pp. 596601,1963.

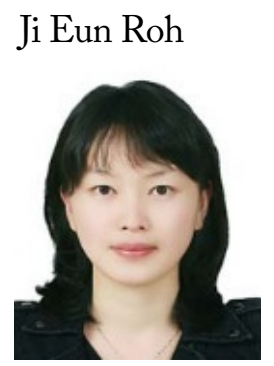

received the Ph.D. degree in Computer Science and Engineering from POSTECH, South Korea, in 2006. Since March, 2006, she has been with the Agency for Defense and Development, South Korea, where she is currently a principal researcher. Her research interests include Airborne AESA radar system, radar resource management, and radar data processing.

Sung Chul Park

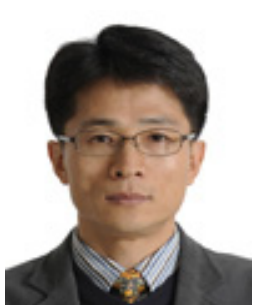

received the M.S. degree in Electronic Engineering from Kwangwoon University, South Korea, in 1990. Since March, 1990, he has been with the Agency for Defense and Development, South Korea, where he is currently a principal researcher. His research interests include radar system design, radar signal processing, and radar data processing. 